Draft Version OCtOBER 20, 2021

Typeset using LATEX default style in AASTeX63

\title{
The X-ray binary population in the Galactic Center revealed through multi-decade observations
}

\author{
Kaya Mori, ${ }^{1}$ Charles J. Hailey, ${ }^{1}$ Theo Y.E. Schutt,${ }^{1}$ Shifra Mandel, ${ }^{1}$ Keri Heuer, ${ }^{2}$ Jonathan E. Grindlay, ${ }^{3}$ \\ Jaesub Hong, ${ }^{3}$ Gabriele Ponti, ${ }^{4,5}$ And John A. Tomsick ${ }^{6}$ \\ ${ }^{1}$ Columbia Astrophysics Laboratory, Columbia University, New York, NY 10027, USA \\ ${ }^{2}$ Department of Physics, Drexel University, 816 Disque Hall, 32 S. 32nd Street Philadelphia, PA 19104, USA \\ ${ }^{3}$ Harvard-Smithsonian Center for Astrophysics, Cambridge, MA 02138, USA \\ ${ }^{4}$ INAF - Osservatorio Astronomico di Brera, via E. Bianchi 46, I-23807 Merate, Italy \\ ${ }^{5}$ Max-Planck-Institut für Extraterrestrische Physik, Giessenbachstrasse, D-85748, Garching, Germany \\ ${ }^{6}$ Space Sciences Laboratory, University of California, Berkeley, CA 94720, USA
}

\begin{abstract}
We present an investigation of the quiescent and transient X-ray binaries (XRBs) of the Galactic Center (GC). We extended our Chandra analysis of the non-thermal X-ray sources, located in the central parsec, from Hailey et al. (2018), using an additional 4.6 Msec of ACIS-S data obtained in 2012-2018. The individual Chandra spectra of the 12 sources fit to an absorbed power-law model with a mean photon index $\Gamma \approx 2$ and show no Fe emission lines. Long-term variability was detected from nine of them, confirming that a majority are quiescent XRBs. Frequent X-ray monitoring of the GC revealed that the 12 non-thermal X-ray sources, as well as four X-ray transients have shown at most a single outburst over the last two decades. They are distinct from the six known neutron star LMXBs in the GC, which have all undergone multiple outbursts with $\lesssim 5$ year recurrence time on average. Based on the outburst history data of the broader population of X-ray transients, we conclude that the 16 sources represent a population of $\sim 240-630$ tightly-bound BH-LMXBs with $\sim 4-12$ hour orbital periods, consistent with the stellar/binary dynamics modelling in the vicinity of Sgr $\mathrm{A}^{*}$. The distribution of the 16 BH-LMXB candidates is disk-like (at 87\% CL) and aligned with the nuclear star cluster. Our results have implications for XRB formation and the rate of gravitational wave events in other galactic nuclei.
\end{abstract}

Keywords: Galaxy: center — X-rays: binaries — X-rays: bursts

\section{INTRODUCTION}

The central parsec of our Galaxy hosts a large population of X-ray binaries (XRBs), as revealed by deep X-ray surveys and long-term monitoring over the last two decades. The discovery of a dozen non-thermal X-ray sources, using 1.4 Msec of Chandra ACIS-I observations, suggests the existence of hundreds of quiescent XRBs in the central parsec (Hailey et al. 2018, H18). As an overabundance of X-ray transients in the GC has been suggested by Muno et al. (2005b), daily Swift/XRT monitoring of a $25^{\prime} \times 25^{\prime}$ region around Sgr A*, dating back to 2006, has detected a dozen X-ray transients within $\sim 20 \mathrm{pc}$ of the GC (Degenaar et al. 2015). These transients include a variety of source types: a transient magnetar (SGR J1745-29) (Mori et al. 2013; Kennea et al. 2013; Rea et al. 2013), six NS-LMXBs, which have been identified through the detection of type I X-ray bursts, and very faint X-ray transients (VFXTs), whose peak X-ray luminosity is below $10^{36} \mathrm{erg} \mathrm{s}^{-1}$ (Degenaar et al. 2012b). Most recently, two X-ray transients were discovered by Swift in 2016, and the follow-up NuSTAR observations suggest that they are outbursting BH-LMXBs (Mori et al. 2019). These LMXBs, observed in quiescent or outbursting states, are more concentrated within a few parsecs from Sgr A*, compared to the magnetic cataclysmic variable $(\mathrm{CV})$ population, which is spread over the central 10 parsec region (Perez et al. 2015; Hailey et al. 2016; Hong et al. 2016; Zhu et al. 2018). 
These observations of XRBs in the GC not only confirm the prediction that a density cusp of compact objects exists near a supermassive BH (Bahcall \& Wolf 1976, 1977; Morris 1993; Miralda-Escudé \& Gould 2000), but can also test the fundamental theory of how XRBs are formed in the nuclei of galaxies. For example, Szölgyén \& Kocsis (2018) suggests that the distribution of isolated $\mathrm{BHs}$ should be disk-like via vector resonance relaxation around the supermassive $\mathrm{BH}$. Generozov et al. (2018) predicts that the XRB distribution should be concentrated in the central parsec as a result of binary formation via tidal captures, a process enhanced by the high stellar density in the proximity of Sgr A*. Further theoretical studies of stellar-mass BH and binary evolution in the Galactic Center reached similar conclusions that the distribution of isolated BHs and binaries should be elongated along the nuclear star cluster (Gruzinov et al. 2020) and highly concentrated within $r \sim 1$ pc around Sgr A* (Tagawa et al. 2020). Most recently, Baumgardt et al. (2018) and Panamarev et al. (2019) performed N-body simulation to study the dynamics and formation of compact objects in the nuclear star cluster (NSC) around Sgr A*. Besides estimating the rate of tidal disruption and gravitational wave events in the GC, the simulations deduced the density profiles of BHs, white dwarfs and other types of stars in the central few parsec region. To examine these theoretical predictions quantitatively, it is vital to identify the XRBs as neutron star (NS) or BH-LMXBs. Detecting more XRBs in the GC will refine their spatial distribution and luminosity function. Ultimately, the number density of BHs and NSs in the GC can be used to normalize the rate of gravitational wave events from stellar remnants in other galactic nuclei (McKernan et al. 2018; Fragione et al. 2019; Tagawa et al. 2020).

Despite the growing number of Galactic X-ray transients detected by Swift and MAXI, it is still difficult to positively identify BH systems solely based on their X-ray properties, since NS and BH binaries share many common spectral and timing signatures during outbursts and in quiescence. Nevertheless, a census of the X-ray outburst history can be used for determining the nature of X-ray transients (Coriat et al. 2012). Besides some outliers and caveats on source detectability by all-sky X-ray monitors (Knevitt et al. 2014; Arur \& Maccarone 2018; Carbone \& Wijnands 2019), NSLMXB transients tend to have short recurrence times of $\sim 5--10$ years between their X-ray outbursts, while a majority of known BH transients in our Galaxy have undergone outbursts only once in the past 50 years (Corral-Santana et al. 2016).

Nearly continuous monitoring of X-ray transients has been carried out only in the past two decades in the GC region. Especially in the central $r<30$ pc region, daily Swift-XRT monitoring over the last 13 years has detected X-ray transients, including VFXTs, down to $L_{X} \sim 10^{34} \mathrm{erg} \mathrm{s}^{-1}$. Unlike the more infrequent monitoring of several globular clusters, the Swift/XRT GC observation program provides the most complete information about the X-ray outburst histories of a concentration of X-ray sources (Carbone \& Wijnands 2019). As a result, it is unambiguously established that all six identified NS-LMXBs within $r \lesssim 50$ pc and all 5 VFXTs within $r<10$ pc (where Swift-XRT is sensitive enough to detect VFXTs) have short recurrence times ( $\lesssim 5$ years and $\lesssim 10$ years, respectively) on average ${ }^{1}$, while other X-ray transients have undergone outbursts only once in the last two decades or longer. Additionally, a comparison between the numbers of quiescent and transient $\mathrm{BH}$ binaries in the GC can be used to determine the recurrence time of BH-LMXBs robustly. This is one of the fundamental but highly uncertain parameters in the disk instability models (Coriat et al. 2012). The X-ray observations of known BH transients in the Galactic plane set only a lower limit of recurrence time to $\sim 50$ years, with some exceptions, mostly of binaries with an evolved donor (e.g., GX 339-4, V404 Cyg, and V4641 Sgr with more frequent X-ray outbursts; Corral-Santana et al. 2016).

This is a follow-up paper to H18, and we investigate the properties of both quiescent and transient XRBs in the GC. Based on our subsequent ACIS-S analysis, we update our spectral and variability studies on the dozen non-thermal X-ray sources discovered by H18 as well as another quiescent XRB (qXRB) candidate (§2). Our ACIS-S analysis further establishes the results of $\mathrm{H} 18$ by fitting individual source spectra more accurately and detecting variability from four more non-thermal X-ray sources (more specifically, we found an additional non-thermal X-ray source, and with improved statistics from the addition of ACIS-S data, one of the original dozen H18 sources appears to be a magnetic CV after further analysis). We compare their X-ray properties with other known BH or NS transients in our Galaxy and argue that the 12 non-thermal sources, two Swift transients in 2016 (Mori et al. 2019) and other X-ray transients with single outbursts (Davies et al. 1976; Muno et al. 2005a) are most consistent with BH-LMXBs (§3). Based on recent correlation studies of X-ray outburst luminosity and recurrence rates, we constrain the orbital period range of the $16 \mathrm{BH}-\mathrm{LMXB}$ candidates and discuss the nature of VFXTs in the GC. In $\S 4$, we investigate the spatial

\footnotetext{
${ }^{1}$ We define the average recurrence time as 20 years (the period of near-constant X-ray monitoring in the GC, starting in 2000) divided by the number of outbursts observed over that period.
} 
distribution of these 16 BH-LMXB candidates, all located in the central few parsec region. In §5, we discuss the spatial distribution, number density and properties of the BH-LMXBs in light of recent theoretical models for XRB formation in the vicinity of Sgr $A^{*}$. While the paper focuses mainly on studying BH-LMXBs in the GC, a large population of quiescent NS-LMXBs may exist in the GC as observed in globular clusters (Heinke et al. 2003). A majority of these sources are undetectable by X-ray telescopes since their dominant soft thermal emission $(k T \lesssim 0.1 \mathrm{keV}$; Degenaar et al. 2012a; Walsh et al. 2015) is heavily obscured by the large hydrogen column density $\left(N_{\mathrm{H}} \sim 10^{23} \mathrm{~cm}^{-2}\right)$ in the GC, and they do not show X-ray outbursts due to their low accretion rates. Finally, we summarize our results in $\S 6$. Throughout the paper, we assume a distance to the GC of $8 \mathrm{kpc}$ (Camarillo et al. 2018).

\section{CHANDRA ACIS ANALYSIS OF NON-THERMAL X-RAY SOURCES IN THE GC}

In this section, we present our follow-up Chandra ACIS analysis of the dozen non-thermal X-ray sources discovered by H18. We extended the analysis by including 38 additional ACIS-S/HETG observations (3 Msec total exposure) from 2012 and 40 ACIS-S observations (1.6 Msec total exposure) from 2013-2018 and combined them with the ACISI data (2002-2011) for the dozen non-thermal X-ray sources published in H18. The improved photon statistics, yielding $~ 300-600$ net counts for most of the non-thermal X-ray sources, allow us to fit individual source spectra to determine their power-law indices rather than using hardness ratios (§2.1). Additionally, the longer time baseline from incorporating ACIS-I, ACIS-S/HETG and ACIS-S data allows us to detect source variability, thus distinguishing between qXRBs and rotation-powered MSPs (§2.2). We did not include ACIS-S observations for one of the non-thermal sources (CXO J174540.38-290033.5) because of the background contamination from the nearby transient magnetar SGR J1745-29.

We used ACIS Extract (AE) software for spectral analysis (Broos et al. 2010). We extracted source photons from a region encompassing $90 \%$ of the local point spread function (typically $\sim 1^{\prime \prime}$ ) around the Chandra position of each source. Background extraction was completed by extracting photons from an annular region centered on the source with a background-to-source region area ratio nominally set to 5 and by avoiding nearby point sources. Response matrices and effective area files were also produced for each observation by AE. We also applied the dust scattering model in XSPEC (Arnaud 1996) to take into account the (energy-dependent) fraction of source photons scattered to outside the extraction region in Chandra data (Jin et al. 2018). Note that the long-term degradation of the ACIS effective area (https://cxc.cfa.harvard.edu/ciao/why/acisqecontamN0010.html) is negligible at $E \gtrsim 2 \mathrm{keV}$, where the GC sources are detected, due to the ISM absorption and scattering of soft photons. Hence, the ACIS contamination does not affect our stacked spectral analysis and variability study. More details can be found in H18.
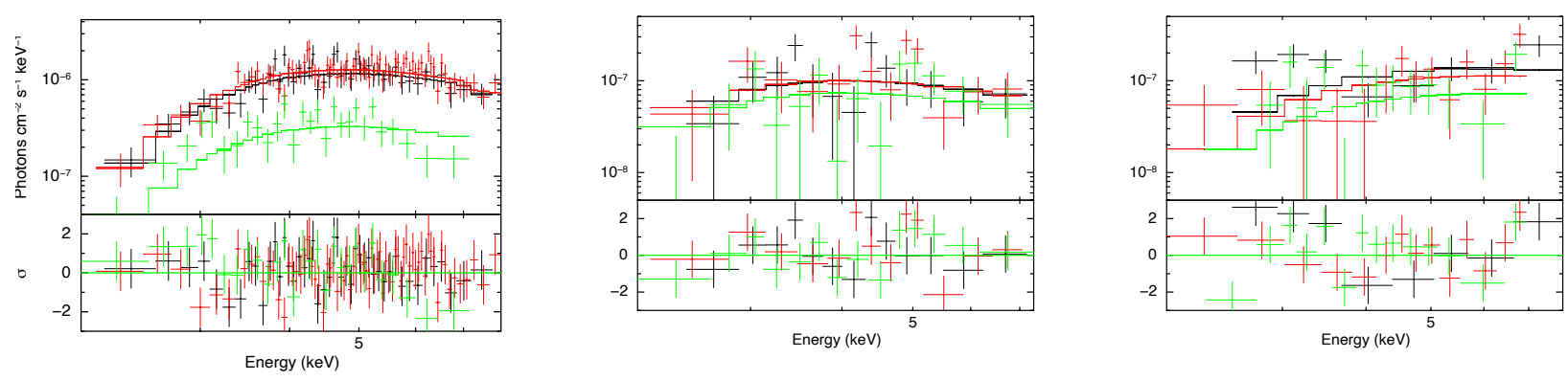

Figure 1. Chandra ACIS-I (black), ACIS-S/HETG (red) and ACIS-S (green) spectra of CXO J174540.16-290055.6 (left) and CXO J174540.37-290049.9 (middle), representing the brightest and faintest of the sources, respectively. Right: ACIS spectra of CXO J174539.28-290049.1, one of the dozen non-thermal sources listed in H18, which is now classified as a thermal source with atomic lines. All ACIS spectra are fitted with an absorbed power-law model; residuals are shown in the lower panels.

\subsection{Spectral analysis}

By combining ACIS-I, ACIS-S/HETG and ACIS-S data, we are able to fit individual spectra in XSPEC and determine their power-law indices more accurately. We rebinned each spectrum with 20 counts per bin and applied chi-squared statistics. Fitting unbinned ACIS spectra with C-statistics yielded similar results. 
As a result, we found another qXRB (CXO J174540.16-290055.6) with a non-thermal X-ray spectrum. This Chandra X-ray source, listed in the Muno et al. (2009) and Zhu et al. (2018) catalogs, is located $\sim 27^{\prime \prime}(\sim 1.1 \mathrm{pc})$ away from Sgr $\mathrm{A}^{*}$. In H18, we tagged it as a marginally soft X-ray source, or qXRB, potentially with a non-thermal spectrum with $\Gamma \gtrsim 1.5$, given its hardness ratio HR2 0.6 (see below for more details). On the other hand, we found that one of the dozen non-thermal sources listed in H18 (CXO J174539.28-290049.1) shows an Fe line feature at $E=6.7 \mathrm{keV}$ when we fit its ACIS spectra with an absorbed power-law model, yielding a hard photon index $\Gamma=0.8_{-0.9}^{+0.8}\left(\chi_{\nu}^{2}=1.9\right.$ for 32 dof). See the right panel of Figure 1. An absorbed thermal APEC model fits the ACIS spectra better with $k T=7_{-3}^{+4} \mathrm{keV}$ with a $\chi_{\nu}^{2}=1.5$ (31 dof). For both models, $N_{\mathrm{H}}$ is fixed to $1.2 \times 10^{23} \mathrm{~cm}^{-2}$, the best-fit column density from the stacked ACIS spectra of the 12 non-thermal sources (see below). Based on the presence of Fe line emission and a plasma temperature consistent with the typical range for CVs (Mukai 2017), we conclude that this source is a thermal X-ray source (likely a CV) and exclude it from further analysis. As we demonstrated with MARX simulations in H18, finding one thermal source out of the dozen non-thermal sources is consistent with the expected rate of false source identification based on the ACIS-I hardness ratio analysis. All other non-thermal X-ray sources from H18 fit well to an absorbed power-law model with $\chi_{\nu}^{2}=0.8-1.1$ (for $\sim 40-150$ dof). We also fit unbinned ACIS spectra using C-statistics and found that the residuals are either insignificant or too narrow (compared to the detector energy resolution), indicating that they are produced by statistical fluctuations.

Based on spectral simulations of mCVs at the GC distance, assuming a two-temperature APEC model with the typical range of plasma temperatures and abundances, we found that $\mathrm{mCVs}$ with $>300$ net counts generally have resolvable Fe line emission and significantly harder continuum emission from thermal bremsstrahlung and cyclotron cooling than non-thermal accretion disk emission (Hailey et al. 2018). One exception is GK Per, an IP with a featureless power-law X-ray spectrum with $\Gamma \sim 2$, whose Fe abundance was observed to be extremely low (e.g., $A_{\mathrm{Fe}} \sim 0.1$ for GK Per; Xu et al. 2016). However, we find the existence of a cusp of peculiar GK Per-like CVs in the central parsec region highly unlikely, given that hundreds of thermal X-ray sources in the GC exhibit much higher (and typical for CVs) Fe abundances of $A_{\mathrm{Fe}}=0.7-0.8$ (Muno et al. 2004). Thus, we conclude that none of the 12 non-thermal sources can be mCVs based on the additional ACIS-S observations. In addition, we considered the possibility of non-magnetic CVs (nmCVs), since a fraction of those are bright in the X-ray band (Baskill et al. 2005). We adopted the spectral parameters obtained from 16 typical nmCVs listed in Xu et al. (2016) and simulated Chandra/ACIS spectra using the actual background spectra of the dozen non-thermal sources. Xu et al. (2016) showed that the mean equivalent widths of Fe lines at 6.4, 6.7 and $7.0 \mathrm{keV}$ are 60, 440 and $100 \mathrm{eV}$, respectively. For completeness, we also considered SS Cyg, which has one of the hardest and brightest X-ray spectra - with weaker Fe lines - among nmCVs. Given the number of ACIS counts from each of the sources $(\gtrsim 1,000)$ and their lack of Fe emission lines, we found that none of the dozen source spectra are consistent with those of typical nmCVs, while one of them could potentially be an SS Cyg-like CV.

Table 1 lists the updated spectral properties of the 12 non-thermal X-ray sources. The range in the $2-8 \mathrm{keV}$ luminosity column reflects the flux variation between the ACIS-I, ACIS-S/HETG and ACIS-S spectra. For example, we present Chandra/ACIS spectra of CXO J174540.16-290055.6 and CXO J174540.37-290049.9 in Figure 1, as examples of bright and faint sources, respectively. For the new non-thermal source (CXO J174540.16-290055.6), its joint ACIS-I, ACIS-S/HETG and ACIS-S spectra fit well to an absorbed power-law model $\left(\chi_{\nu}^{2}=0.99\right.$ for 142 dof $)$ with $\Gamma=2.1 \pm 0.2$ and $N_{\mathrm{H}}=\left(18_{-1}^{+2}\right) \times 10^{22} \mathrm{~cm}^{-2}$ (the left panel in Figure 1). Its $2-8 \mathrm{keV}$ unabsorbed luminosity is $3 \times 10^{32} \mathrm{erg} \mathrm{s}^{-1}$. Fitting to an absorbed thermal APEC model, representing typical magnetic CV spectra in the Chandra band, led to $N_{\mathrm{H}}=(16 \pm 1) \times 10^{22} \mathrm{~cm}^{-2}, k T=7.6 \pm 1 \mathrm{keV}$ and $A_{\mathrm{Fe}}<0.04$ (90\% C.L.) ( $\chi_{\nu}^{2}=0.99$ for 142 dof). The lack of Fe emission lines, as evident from $A_{\mathrm{Fe}} \approx 0$, establishes that this is a non-thermal X-ray source, not a CV.

Subsequently, we stacked ACIS-I, ACIS-S/HETG and ACIS-S spectra of the 12 non-thermal sources and jointly fit them with an absorbed power-law model by allowing flux normalization factors to vary between the three ACIS spectra (see Figure 2). The best-fit parameters are $N_{\mathrm{H}}=(1.2 \pm 0.2) \times 10^{23} \mathrm{~cm}^{-2}$ and $\Gamma=1.9 \pm 0.2\left(\chi_{\nu}^{2}=1.06 ; 234\right.$ dof). The lack of Fe lines in the stacked ACIS spectra rules out mCVs whose Fe line EWs exceed $\sim 300 \mathrm{eV}$ (Xu et al. 2016). Moreover, $\mathrm{Xu}$ et al. (2019) found that thermal X-ray sources in the diffuse hard X-ray emission region within the central $10 \mathrm{pc}$ show strong Fe lines with their total EW above $\sim 500 \mathrm{eV}$. Clearly, the dozen non-thermal sources exhibit no such strong Fe lines in their individual and stacked ACIS spectra.

\subsection{Source variability}




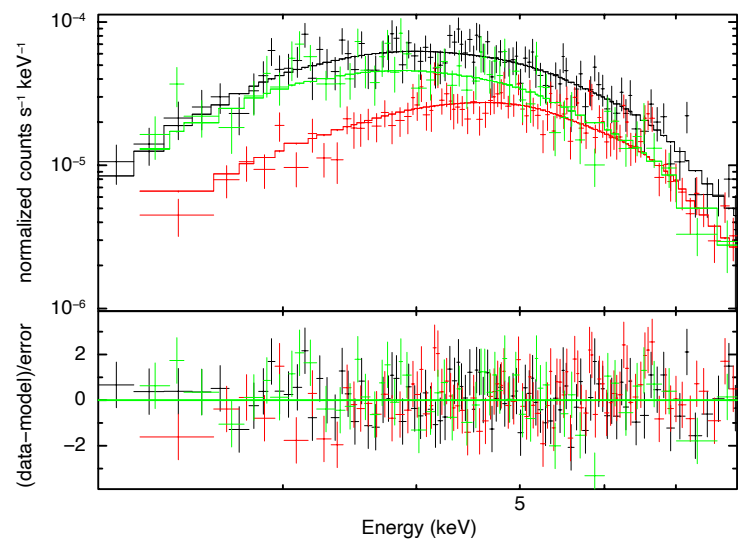

Figure 2. Stacked Chandra ACIS-I (black), ACIS-S/HETG (red) and ACIS-S (green) spectra of the 12 non-thermal sources jointly fit with an absorbed power-law model. The residuals are shown in the lower panel.

Source variability is a robust diagnostic to distinguish between LMXBs and rotation-powered millisecond pulsars (rMSPs). Many LMXBs in the quiescent state have shown evidence of X-ray flux variability, by a factor of $2-5$ over a timescale of days to years, due to varying accretion rate (Plotkin et al. 2013). While rMSPs also have non-thermal X-ray emission, they display no such long term variability (Bogdanov et al. 2006).

We investigated the source variability in three different approaches as listed in Table 1 - Bayesian Block (BB) analysis (Scargle et al. 2013), Kolmogorov-Smirnov (KS) test, and flux variation between the ACIS-I (2002-2011), ACIS-S/HETG (2012) and ACIS-S (2013-2018) spectra. Following H18, we first combined individual event files

Table 1. Spectral and timing properties of the 12 non-thermal X-ray sources

\begin{tabular}{|c|c|c|c|c|}
\hline Source name & Photon index & $L_{\mathrm{X}}\left[10^{31} \mathrm{erg} \mathrm{s}^{-1}\right]$ & Variability significance $[\sigma]^{a}$ & Variability detection methods $b$ \\
\hline $174539.87-290034.2$ & $2.2_{-0.7}^{+0.8}$ & $8.1-30$ & 12.8 & BB (ACIS-I), FV, KS (ACIS-S) \\
\hline $174540.38-290033.5$ & $1.7_{-1.8}^{+1.5 c}$ & $0.3-4.9$ & 3.8 & $\mathrm{FV}$ \\
\hline $174540.40-290024.1$ & $2.0_{-0.7}^{+0.8}$ & $7.9-16$ & 4.5 & $\mathrm{FV}$ \\
\hline $174540.45-290036.3$ & $1.5_{-0.8}^{+0.7 c}$ & $4.0-6.8$ & 2.5 & BB (ACIS-I), FV \\
\hline $174540.79-290024.5$ & $2.8_{-1.0}^{+1.2}$ & $4.7-18$ & 7.2 & BB (ACIS-I), FV \\
\hline $174539.40-290040.9$ & $2.9_{-0.9}^{+1.0}$ & $8.2-9.8$ & 1.6 & BB (ACIS-I) \\
\hline $174540.95-290031.2$ & $2.1 \pm 0.7^{c}$ & $2.0-6.2$ & 3.8 & $\mathrm{FV}$ \\
\hline $174541.03-290026.8$ & $1.7_{-0.8}^{+0.7 c}$ & $3.3-5.2$ & 1.6 & FV, KS (ACIS-I) \\
\hline $174540.63-290013.4$ & $2.1_{-0.8}^{+0.9}$ & $2.3-15$ & 12.1 & FV, KS (ACIS-S/HETG) \\
\hline $174539.48-290045.8$ & $2.9_{-0.5}^{+0.6}$ & $6.3-30$ & 19.5 & $\mathrm{BB}$ (ACIS-I), FV \\
\hline $174540.37-290049.9$ & $2.2 \pm 0.5^{c}$ & $3.1-4.3$ & 1.4 & $\mathrm{FV}$ \\
\hline $174540.16-290055.6$ & $2.0 \pm 0.4$ & $20-77$ & 24.0 & BB (All), FV, KS (All) \\
\hline
\end{tabular}

Note $-L_{\mathrm{X}}$ for each source indicates a range of unabsorbed $2-8 \mathrm{keV}$ luminosity measured from ACIS-I and ACIS-S spectra.

${ }^{a}$ We listed the highest significance of variability detection among the three methods applied to each source.

${ }^{b}$ We listed the methods which detected source variability at $>90 \%$ significance. BB, KS and FV stand for Bayesian Block analysis, KS test and flux variation between ACIS-I, ACIS-S/HETG and ACIS-S spectra, respectively.

${ }^{c}$ The power-law index was not well constrained when $N_{\mathrm{H}}$ was fit freely. We fixed $N_{\mathrm{H}}$ to $1.2 \times 10^{23} \mathrm{~cm}^{-2}$, which is the best-fit column density from the stacked ACIS spectra of the 12 non-thermal sources. 


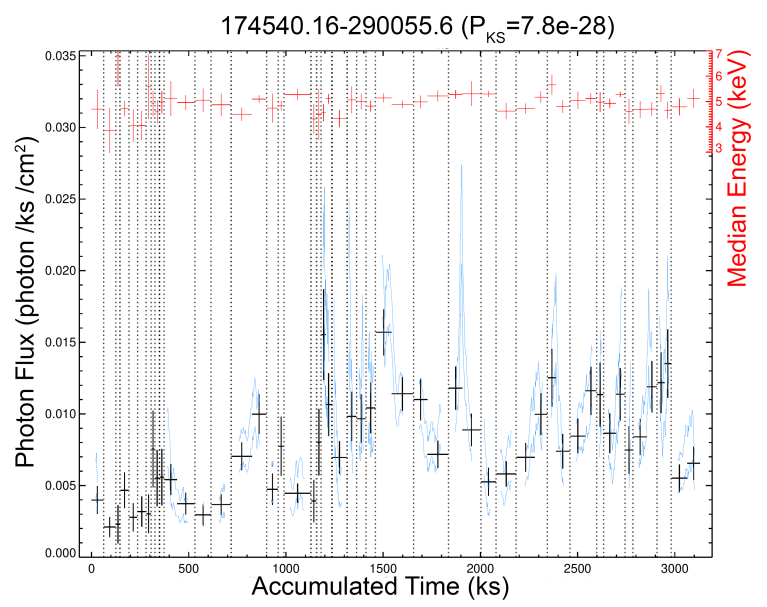

Figure 3. Chandra ACIS-S sequenced lightcurve of CXO J174540.16-290055.6, with gross flux in time bins of constant significance (black), median energy of the events in each bin (red), and pointwise $68 \%$ confidence band for the continuous lightcurve (blue). Each observation is separated by a vertical dashed line.

in each of the ACIS-I, ACIS-S/HETG and ACIS-S data sets separately, then applied the BB and KS tests to the unbinned photon arrival times in each merged event file. We found that $7(2)$ sources were variable at $>90 \%(>99 \%)$ confidence level (CL), including the new non-thermal source CXO J174540.16-290055.6. Neither BB nor KS tests detected significant variability in the ACIS-S/HETG and ACIS-S data of the other non-thermal sources. As presented in §2.1, we jointly fit the ACIS-I, ACIS-S/HETG and ACIS-S spectra for each source with an absorbed power-law model and calculated the flux normalization factor between the three spectra. Nine out of the 12 non-thermal Xray sources showed X-ray flux variation above 90\% CL. For example, in Figure 3, Chandra 2-8 keV lightcurves of CXO J174540.16-290055.6 show that the source is highly variable with KS test p-value $=8 \times 10^{-28}$. The source variability rules out the possibility that this source is a rMSP. We also note that none of the 12 non-thermal sources have had X-ray outbursts $\left(L_{X} \gtrsim 10^{34} \mathrm{erg} \mathrm{s}^{-1}\right)$ detected, despite the frequent GC monitoring over the past two decades.

\section{X-RAY BINARIES IN THE GC, THEIR OUTBURST HISTORY AND QUIESCENT STATES}

Unlike the detection of type I X-ray bursts or pulsations from NS transients, there is no "smoking gun" for identifying $\mathrm{BH}$ transients solely based on their X-ray properties, with a few exceptions. Broad Fe emission lines, often detected from X-ray transients, can result from relativistic effects near a fast-spinning BH. A high spin value above $a_{*} \sim 0.7$, measured through broad-band X-ray spectral fitting, establishes the BH transient case (Miller \& Miller 2015), since observed spin values for NS transients are much smaller - e.g., $a_{*}=0.15$ for the NS-LMXB 4U 1728-34 (Sleator et al. 2016). Detection of a bright radio jet during the low/hard state has also been considered a diagnostic for BH transients, since the radio jets from outbursting NS-LMXBs are fainter by a factor of $\sim 22$ (Gallo et al. 2018). On the other hand, it is difficult to definitively classify the variable, non-thermal X-ray sources we discussed in $§ 2$ as NSor BH-LMXBs, since in quiescence, many of their spectral and timing properties are similar. This leaves open the question of whether the non-thermal X-ray sources are NS- or BH-LMXBs.

In this section, we review (1) recent studies on the X-ray outburst history of NS/BH LMXBs (§3.1) and (2) the $\mathrm{X}$-ray transients discovered in the GC and their outburst history ( $§ 3.2)$. Based on the most recent, unbiased analysis of X-ray outbursts, we argue that the dozen non-thermal X-ray sources are consistent with BH-LMXBs (§3.3). Their quiescent X-ray spectra, obtained with our Chandra ACIS analysis, provide further support for the BH-LMXB scenario (§3.3.1). We also discuss the nature of VFXTs, which may belong to a different class of LMXBs, in the GC (§3.4).

\subsection{X-ray outburst history of NS and BH LMXBs}

Recently, it has become more evident that X-ray outburst history can be used as a diagnostic tool to infer the nature of LMXBs (Yan \& Yu 2015; Lin et al. 2019). Except for several outliers (e.g., Cen X-4), ${ }^{2}$ most NS transients

\footnotetext{
${ }^{2}$ Two X-ray outbursts were detected from Cen X-4 in 1969 and 1979 (Conner et al. 1969; Matsuoka et al. 1980). Since then, no X-ray outburst has been detected from this NS-LMXB.
} 
have a short recurrence time of $\sim 5-10$ years between their X-ray outbursts Coriat et al. (2012). On the other hand, a majority $(73 \%)$ of known BH transients in our Galaxy have had only one outburst in the past $\sim 50$ years (CorralSantana et al. 2016). Hereafter, for clarity, we refer to X-ray transients with only one detected outburst as single X-ray outburst transients (SXOTs). In the scheme of the disk instability model (DIM), Coriat et al. (2012) offered a plausible explanation for the distinct outburst recurrence trends between NS- and BH-LMXBs to the "transientness" parameter (i.e. a ratio of the estimated mass transfer rate $\dot{M}$ to a critical accretion rate $\dot{M}_{c r}$ ), barring some uncertainties on the $\dot{M}$ measurements and ambiguous source types on whether they contain NS or BHs.

In general, the poor detectability of faint X-ray transients through all-sky monitoring with Swift-BAT and MAXI hampers the accurate measurement of recurrence times. Overall, VFXTs and most X-ray transients at large distances are subject to this selection effect with all-sky X-ray monitoring. Even though several globular clusters (e.g. NGC 6388, Terzan 5) have been monitored by Swift/XRT, Carbone \& Wijnands (2019) demonstrated that their infrequent observing cadence may lead to missing multiple X-ray outbursts and VFXTs for a range of duty cycles and outburst durations. For instance, 47 Tuc, a globular clusters that is among the most extensively observed by Swift/XRT, has had $\lesssim 200$ ksec total exposure time, whereas the ongoing Swift/XRT GC monitoring program has accumulated several Msec worth of exposures since 2006 (Degenaar et al. 2015). In addition, source identification may be difficult due to the high background X-ray emission and source confusion in globular clusters (e.g., if a transient is not quickly followed up by Chandra during its outburst). Carbone \& Wijnands (2019) performed Monte-Carlo simulation for assessing the Swift/XRT detectability of X-ray transients and determined that the Swift/XRT monitoring of the GC offers the only nearly complete survey of X-ray outbursts down to the luminosity range of VFXTs $\left(L_{X} \sim 10^{34} \mathrm{erg} \mathrm{s}^{-1}\right) .{ }^{3}$ However, this observation bias primarily affects NS transients, since BH-LMXB outbursts tend to be both significantly brighter and longer in duration than their NS counterparts (Yan \& Yu 2015). Therefore, NS-LMXB outbursts are much more likely to go undetected, indicating that the outburst frequency of NS transients may be even higher than currently known.

This makes it highly unlikely that most of the 12 non-thermal GC sources are NS-LMXBs; due to the near-constant $\mathrm{X}$-ray monitoring of the GC region over the past two decades, any outbursts from these sources would likely have been detected (H18). While persistently quiescent NS-LMXBs do exist - many such sources have been identified in globular clusters - those binaries are believed to undergo little to no accretion; their X-ray emission is predominantly thermal and non-varying, as it originates from the NS surface (Bogdanov et al. 2016). Such an origin can be excluded for our 12 non-thermal sources. In globular clusters, only a few confirmed NS-LMXBs (e.g. CX3 in Terzan 5) are known to have significant non-thermal components in their X-ray spectra above $E \sim 2 \mathrm{keV}$ (Bahramian et al. 2020), largely due to their low accretion rates (a result of aging binary populations) and the lack of extensive Chandra observations (unlike the GC with $\sim 7 \mathrm{Msec}$ total exposure as presented in this paper). Furthermore, the stellar populations and environments of globular clusters are distinct from those in the Galactic disk and GC (Heinke et al. 2003). Therefore, hereafter we adopt the historical data of GC transients collected over the last two decades as the most reliable sample for identifying the dozen non-thermal sources in the central parsec.

\subsection{X-ray transients in the $G C$}

In 2006 February, Swift initiated a daily XRT monitoring program for detecting X-ray flares from Sgr A* and X-ray transients in the central $25^{\prime} \times 25^{\prime}(60 \mathrm{pc} \times 60 \mathrm{pc})$ region. Since then, Swift has detected a dozen X-ray transients (including some recurrent transients discovered earlier) within $\sim 50 \mathrm{pc}$ of the GC (Degenaar et al. 2015), including a new transient magnetar (Mori et al. 2013). We compiled all the X-ray transients detected in the central $\sim 50 \mathrm{pc}$ region where X-ray telescopes such as Chandra, XMM-Newton and Swift are sensitive to detecting X-ray outbursts above $\sim 10^{34} \mathrm{erg} \mathrm{s}^{-1}$. In total, there have been $20 \mathrm{X}$-ray transients detected in the GC.

Besides the transient magnetar SGR J1745-2900, these GC transients can be classified into three groups: six NSLMXBs, four SXOTs and 9 VFXTs. The six NS-LMXBs have been identified through type I X-ray bursts and/or pulsation detection (Degenaar et al. 2012b). The four SXOTs include the two Swift transients detected in 2016 (SWIFT J174540.7-290015 and SWIFT J174540.2-290037), CXOGC J174540.0-290031 and 1A 1742-289. CXOGC J174540.0-290031 was detected by Chandra in 2005 (Muno et al. 2005a). Follow-up NuSTAR observations suggest that the 2016 Swift transients are BH-LMXBs due to their high BH spin values of $a_{*}>0.9$ (Mori et al. 2019).

${ }^{3}$ Carbone \& Wijnands (2019) pointed out that an X-ray transient in the GC may remain undetected if its outburst is periodic and somehow coincides with the annual Swift/XRT observation gap that occurs from November to February. However, we consider such a case to be very unlikely, given the typically stochastic nature of X-ray outburst recurrence time and average outburst duration. 


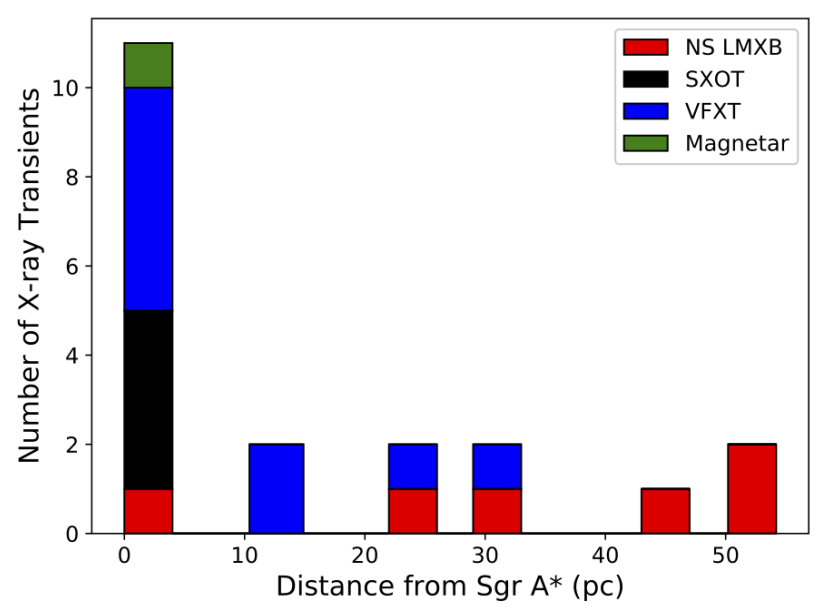

Figure 4. Radial distribution of all the X-ray transient sources detected within $r \sim 50$ pc of the GC. The number of transients is plotted at different projected distances [pc] from Sgr A*. 5 of the 9 VFXTs are located within $<10$ pc and recurrent X-ray outbursts have been detected from them by Swift/XRT. 4 other VFXTs, all of which are outside $\sim 10$ pc, have been detected only once.

An outburst from 1A 1742-289, located at $\sim 2$ pc from Sgr A*, was detected in the radio and X-ray bands in 1975 (Davies et al. 1976). Its radio position distinguished the source from nearby X-ray transients such as AX J1745.6-2901. Bright radio jets in the range of $L_{\text {radio }} \sim 10^{32}-10^{33} \mathrm{erg} \mathrm{s}^{-1}$ were observed from both CXOGC J174540.0-290031 and 1A $1742-289$ during their X-ray outbursts. Following the well-established $L_{\text {radio }}$ vs $L_{\mathrm{X}}$ correlation in the hard state (Gallo et al. 2018), the radio-loud outbursts suggest that they are BH transients. Hence, all four SXOTs are likely $\mathrm{BH}$ transients, which is consistent with the fact that a majority of known BH transients in the Galactic disk have long recurrence times (Corral-Santana et al. 2016). For the VFXTs in the GC, it is still unknown whether they harbor a NS or BH. As shown in Figure 4, about half of all the GC X-ray transients detected by Swift, Chandra and $X M M-N e w t o n$ are concentrated in the central few parsecs around Sgr A*. All four SXOTs (BH transients) are located at $r \lesssim 2$ pc, while the NS-LMXBs and VFXTs are distributed more widely beyond $r \sim 10 \mathrm{pc}$.

As mentioned above, the most frequent and sensitive survey of X-ray transients, down to $L_{\mathrm{X}} \sim 10^{34} \mathrm{erg} \mathrm{s}^{-1}$, has been carried out in the GC region (Degenaar et al. 2015). In the central $r \lesssim 30$ pc region, daily Swift-XRT monitoring of the GC over the last 13 years is believed to have revealed most X-ray transients down to $L_{X} \sim 10^{34} \mathrm{erg} \mathrm{s}^{-1}$ (Degenaar et al. 2015). Within the central $\sim 50 \mathrm{pc}$ region, all six identified NS-LMXBs have short recurrence times of $\lesssim 5$ years, and five of the nine VFXTs have average recurrence times of $\lesssim 10$ years. Note that Swift-XRT may have missed faint/short outbursts from the four non-recurrent VFXTs (like a two-week long $L_{X} \sim 10^{35} \mathrm{erg} \mathrm{s}^{-1}$ outburst detected from SWIFT J174553.7-290347 at $r \sim 25 \mathrm{pc}$ ), all of which are located outside $10 \mathrm{pc}$ where the XRT sensitivity decreases rapidly (Degenaar \& Wijnands 2009).

Given the lack of detection of X-ray outbursts in the last two decades, the 12 quiescent, non-thermal X-ray sources, along with the four SXOTs (all of which are likely BH transients), are clearly distinct from the 6 known NS-LMXBs and 5 VFXTs within $r<10 \mathrm{pc}$, which have all had recurrent X-ray outbursts. It is also evident that the SXOTs are not VFXTs as their peak X-ray luminosities ${ }^{4}$ exceed $L_{X} \sim 10^{36} \mathrm{erg} \mathrm{s}^{-1}$.

\subsection{Nature of single X-ray outbursters and quiescent XRBs in the GC}

Besides the GC transient monitoring described above, some recent studies on X-ray outburst history can be used to further infer the nature of the SXOTs and quiescent XRBs in the GC. It is well established that peak X-ray outburst luminosity and recurrence time are related to the physical size of the accretion disk and Roche lobe, and thus to the orbital period, as supported by X-ray observations (Wu et al. 2010; Lin et al. 2019). In general, XRBs with shorter orbital periods show fainter X-ray outbursts. Wu et al. (2010) found an empirical relation between the peak $L_{X}$ and

${ }^{4}$ Muno et al. (2005a) estimated the peak X-ray luminosity of CXOGC J174540.0-290031 exceeded $\sim 2 \times 10^{36} \mathrm{erg} \mathrm{s}^{-1}$ based on the scattered $\mathrm{X}$-ray flux from nearby diffuse X-ray sources. 
$P_{\text {orb }}$ [hours] as $\log \left(L_{\mathrm{X}} / L_{\mathrm{Edd}}\right)=-1.80+0.64 \log P_{\mathrm{orb}}$, where $L_{\mathrm{Edd}}$ is the Eddington luminosity. Note that, for a given $P_{\text {orb }}$, BH-LMXBs should (on average) be brighter than NS-LMXBs during X-ray outbursts due to their higher $L_{\text {Edd }}$.

On the other hand, Lin et al. (2019) found a robust relationship between $P_{\text {orb }}$ and recurrence time $\left(\tau_{\text {rec }}\right)$ for bright Xray outbursts $\left(L_{\mathrm{X}} \gtrsim 10^{37} \mathrm{erg} \mathrm{s}^{-1}\right.$ at $\left.8 \mathrm{kpc}\right)$. Their analysis of Swift/BAT, RXTE/ASM and MAXI all-sky monitoring data showed that short-period LMXBs ( $P_{\text {orb }} \lesssim 12$ hours), whether they contain a NS or BH, have undergone only one bright outburst $\left(L_{X} \gtrsim 10^{36} \mathrm{erg} \mathrm{s}^{-1}\right)$ over more than a decade, whereas multiple such outbursts have been observed with $\tau_{\text {rec }}<10$ years from long-period LMXBs ( $P_{\text {orb }} \gtrsim 12$ hours). Lin et al. (2019) attributed this distinct outbursting behavior to the critical orbital periods ( $P_{\text {orb }}=12.4$ and 12.7 hours for NS-LMXBs and BH-LMXBs, respectively), above which they can harbor an evolved donor, yielding higher accretion rates, and thus shorter $\tau_{\text {rec }}$. However, this relation between $P_{\text {orb }}$ and $\tau_{\text {rec }}$ is more complicated for NS-LMXBs by some selection effects, while it is more robust for BH-LMXBs, as we describe below.

NS-LMXBs and recurrent transients - As Lin et al. (2019) pointed out, fainter X-ray outbursts may have gone undetected by all-sky monitors, and thus can bias the X-ray outburst recurrence data. This selection effect is more significant for NS-LMXBs, since the lower $L_{\text {Edd }}$ yields smaller peak X-ray luminosity for a given $P_{\text {orb }}$, as apparent in the Wu et al. (2010) formula. As a result, the relation between $P_{\text {orb }}$ and $\tau_{\text {rec }}$ is not reliable for NS-LMXBs with $P_{\text {orb }} \lesssim 20$ hrs (i.e. most of the NS-LMXB sources sampled by Lin et al. (2019)) since some of their X-ray outbursts are likely too faint to be detected by all sky monitoring. This implies that a larger number of (faint) X-ray outbursts from NS-LMXBs with $P_{\text {orb }} \lesssim 20$ hrs could have gone undetected by all-sky monitors (Lin et al. 2019) - this is particularly true for NS-LMXBs at large distances such as those in the GC.

However, the outburst history of X-ray transients in the GC has been robustly determined by frequent X-ray monitoring over the last two decades - all six NS-LMXBs within $\sim 50$ pc of the GC have short recurrence time ( $\lesssim 5$ years) on average. A good example is AX J1745.6-2901, with an $8.4 \mathrm{hr}$ orbital period (Ponti et al. 2017), which has shown multiple X-ray outbursts in the last decade. Therefore, we conclude that the quiescent XRBs in the GC are most likely not NS-LMXBs since no X-ray outbursts have been detected from any of them over the last two decades. We emphasize that this argument is robust as it is based solely on the unbiased historical data of the X-ray transients in the GC.

BH-LMXBs and single X-ray outbursters - In contrast to the NS-LMXB case, Lin et al. (2019) argued that the relation between $P_{\text {orb }}$ and $\tau_{\text {rec }}$ is robust for local BH-LMXBs since their relatively brighter X-ray outbursts are not subject to the same selection effects, except for ultra-compact BH-LMXBs with $P_{\text {orb }} \lesssim 0.9$ hrs (which are likely VFXTs as mentioned below). All BH-LMXBs with $P_{\text {orb }}<12$ hours listed by Lin et al. (2019) are SXOTs, aside from the BHLMXB XTE J1118+480 with $P_{\text {orb }}=4.08$ hours. Note that XTE J1118+480 was marginally considered a VFXT, since its peak X-ray luminosity barely exceeded $L_{\mathrm{X}}=10^{36} \mathrm{erg} \mathrm{s}^{-1}$ (i.e. the conventional threshold between VFXTs and "regular" X-ray transients) and that its orbital period is just above the period gap (Maccarone \& Patruno 2012). XTE J1118+480 is an outlier in several respects and likely belongs to a unique class of BH-VFXTs. Thus, we conclude that the 12 non-thermal X-ray sources, with no X-ray outbursts detected in the past two decades, are likely BHLMXBs with $P_{\text {orb }} \lesssim 12 \mathrm{hrs}$. Additionally, as three of the four SXOTs in the GC have the peak $L_{X} \gtrsim 10^{37} \mathrm{erg} \mathrm{s}^{-1}$ corresponding to $L_{X} / L_{\mathrm{Edd}} \gtrsim 1 \%$ (for a $10 M_{\odot} \mathrm{BH}$ ), their orbital periods should be longer than $P_{\text {orb }} \sim 4$ hrs, following the $\mathrm{Wu}$ et al. (2010) formula given above. Indeed, one of the SXOTs (CXOGC J174540.0-290031, whose outburst occurred in 2005) has a $7.9 \mathrm{hr}$ orbital period (Porquet et al. 2005).

\subsubsection{Quiescent X-ray emission of the dozen non-thermal X-ray sources and the single X-ray outburst transients}

Our follow-up analysis of the 2012 ACIS-S/HETG data, presented in $\S 2$, firmly establishes that the 12 X-ray sources emit non-thermal X-rays and that at least 9 of them are variable, indicating that a majority are qXRBs. The combined ACIS-I and ACIS-S spectra for the individual sources, replacing the hardness ratios (H18), yield a more accurate measurement of the photon indices. The mean of their photon indices, listed in Table 1 , is $\Gamma=2.2 \pm 0.2$, which also matches with the best-fit photon index determined from the stacked Chandra ACIS-I spectra of the non-thermal $\mathrm{X}$-ray sources in H18. This is consistent with the mean photon index $(\Gamma \approx 2)$ of quiescent BH-LMXB sources in the Galactic Plane (Plotkin et al. 2013; Reynolds et al. 2014).

Armas Padilla et al. (2014) and originally Garcia et al. (2001) found that BH-LMXBs are generally fainter than NS-LMXBs in quiescence, despite some dependence on the orbital period, distance uncertainty, and some contribution from soft thermal emission in the case of NS-LMXBs. According to Armas Padilla et al. (2014), in the range of 


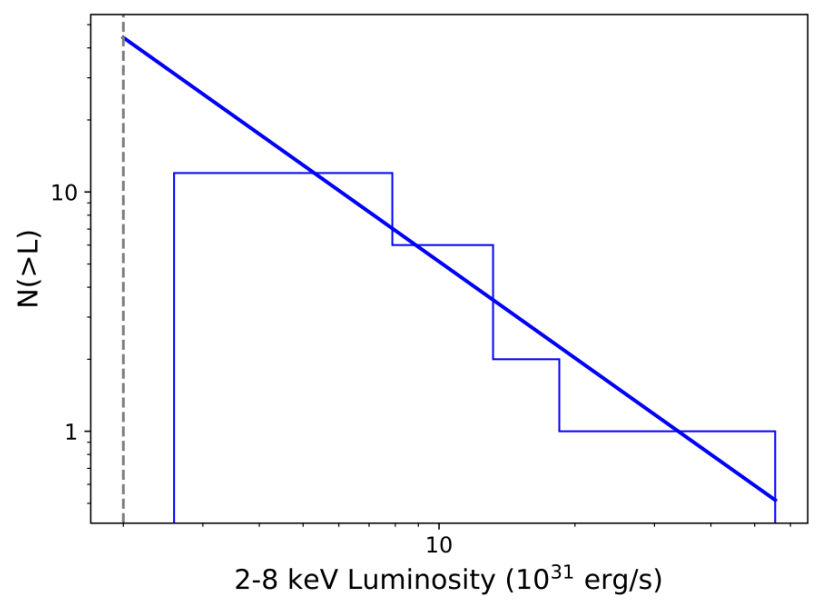

Figure 5. 2-8 keV luminosity function of the 12 non-thermal sources. We adopted the mean $L_{\mathrm{X}}$ value for each source listed in Table 1. The luminosity function is fit by a power-law model $\left(N\left(>L_{\mathrm{X}}\right) \propto L_{\mathrm{X}}^{-\alpha}\right)$ with $\alpha=1.3 \pm 0.1$. The upper limit of quiescent $L_{\mathrm{X}}$ for the two Swift transients (which are likely outbursting BH-LMXBs) detected in 2016 is shown as a dashed vertical line at $L_{\mathrm{X}}=2 \times 10^{31} \mathrm{erg} \mathrm{s}^{-1}$.

$P_{\text {orb }} \approx 4-12$ hours, BH-LMXBs and NS-LMXBs have $L_{X} \sim 1 \times 10^{30}-4 \times 10^{31} \mathrm{erg} \mathrm{s}^{-1}$ and $L_{X} \sim 10^{32}-10^{34} \mathrm{erg} \mathrm{s}^{-1}$, respectively. After converting the $2-8 \mathrm{keV}$ luminosity values listed in Table 1 to the $0.5-10 \mathrm{keV}$ band, the $L_{\mathrm{X}}$ range of the 12 non-thermal sources $\left(5.6 \times 10^{30}\right.$ to $1.6 \times 10^{33} \mathrm{erg} \mathrm{s}^{-1}$, with the mean value at $\left.2.8 \times 10^{32} \mathrm{erg} \mathrm{s}^{-1}\right)$ falls somewhere between those of the BH-LMXBs and NS-LMXBs compiled by Armas Padilla et al. (2014). Figure 5 shows the 2-8 keV luminosity function of the 12 non-thermal sources fit by a power-law model. Given some source variability, we adopted the mean values between the ACIS-I, ACIS-S/HETG and ACIS-S observations using the luminosity data listed in Table 1. We determined the best-fit slope $\alpha=1.3 \pm 0.1$ where $N\left(>L_{X}\right) \propto L_{X}^{-\alpha}$. Fitting to only the 9 sources with variability over $90 \%$ CL yields $\alpha=1.2 \pm 0.1$.

However, there are several reasons why the 12 sources may well represent a brighter tail of the intrinsic luminosity distribution. Firstly, there are other, fainter X-ray sources in the central pc which have not yet been spectroscopically identified due to their lower X-ray counts ; we have only analyzed the brighter among the X-ray sources. Typically, we need $\gtrsim 100$ Chandra ACIS counts $(2-8 \mathrm{keV})$ for hardness ratio analysis and $\gtrsim 300$ counts for spectral fitting in order to distinguish non-thermal X-ray sources from CVs (H18). Secondly, there are local, quiescent BH-LMXBs at luminosities below the faintest of the 12 non-thermal sources, as shown in Armas Padilla et al. (2014), indicating that

Table 2. Classification of LMXBs in the GC

\begin{tabular}{lccccc}
\hline \hline \multicolumn{1}{c}{ Source } & \# of sources & Location & Recurrence time & Compact object type & Orbital period range \\
\hline Non-thermal sources & 12 & $r \lesssim 1 \mathrm{pc}$ & $\gtrsim 13 \mathrm{yrs}$ & $\mathrm{BH}^{a}$ & $\mathrm{BH}^{a}$ \\
SXOTS (single X-ray outbursters) & 4 & $r \lesssim 2 \mathrm{pc}$ & $\gtrsim 13 \mathrm{yrs}$ & $\mathrm{NS}$ & $8.9 \mathrm{hrs}{ }^{c}$ or $\sim 4-12 \mathrm{hrs}{ }^{a}$ \\
NS-LMXBs & 6 & $r \gtrsim 3 \mathrm{pc}$ & $\lesssim 5 \mathrm{yrs}$ & otherwise unknown \\
Recurrent VFXTs & 5 & $r<10 \mathrm{pc}$ & $\lesssim 10 \mathrm{yrs}$ & Unknown & Unknown \\
VFXTs with single outbursts & 4 & $r \gtrsim 10 \mathrm{pc}$ & $\gtrsim 10 \mathrm{yrs}$ & Unknown & Unknown \\
\hline
\end{tabular}

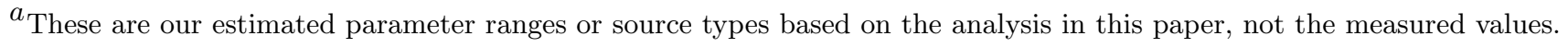

${ }^{b}$ It is possible that only single outbursts have been detected because faint X-ray outbursts may have been missed by Swift/XRT due to the large off-axis distances. The actual recurrence time could be shorter like those VFXTs at $r<10 \mathrm{pc}$.

${ }^{c}$ CXOGC J174540.0-290031 (Porquet et al. 2005).

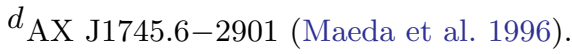


BH-LMXBs of similarly low X-ray luminosities may lie undetected at the GC. Thirdly, some X-ray transients have not been detected in quiescence and their $L_{\mathrm{X}}$ upper limits are much lower than those of the 12 sources. For example, we determined that $L_{X} \lesssim 2 \times 10^{31} \mathrm{erg} \mathrm{s}^{-1}$ in quiescence for the two Swift transients detected in 2016 (Mori et al. 2019). Given that the true luminosity distribution of this source population should be skewed to a lower $L_{X}$ range for the above reasons (and our assumption that the 12 non-thermal sources are representative of the same population), we argue that the underlying source population of the 12 non-thermal sources and the four SXOTs is more consistent with the luminosity distribution of BH-LMXBs. In summary, the distribution of the photon indices and quiescent $L_{\mathrm{X}}$ of the 12 sources supports the BH-LMXB scenario, in addition to the X-ray outburst history discussed in $\S 3.1$. All of the source types and their classifications discussed above are listed in Table 2.

\subsection{Nature of VFXTs in the Galactic Center}

It is generally accepted that VFXTs have smaller accretion rates that produce fainter X-ray outbursts in the range of $L_{X}=10^{34}-10^{36} \mathrm{erg} \mathrm{s}^{-1}$. There are two plausible candidates for the VFXT source type. NS-LMXBs with strong magnetic fields can inhibit accretion flow via propeller effects, thus reducing the mass accretion significantly (Heinke et al. 2015; van den Eijnden et al. 2018). Alternatively, ultra-compact XRBs with very short orbital periods $\left(P_{\text {orb }} \lesssim 1\right.$ hour) can accommodate only hydrogen poor companions due to the small binary separation, and thus the smaller Roche lobe size leads to much weaker accretion flow (Hameury \& Lasota 2016). By applying the DIM to hydrogen poor companions, Hameury \& Lasota (2016) showed theoretically that ultra-compact XRBs can produce recurrent, faint, short-duration $(\sim$ weeks) X-ray outbursts over a few years. These outburst features in the DIM match with those of the VFXTs in general. As Maccarone \& Patruno (2012) pointed out, some VFXTs may be LMXBs in the so-called period gap, with 2-3 hour orbital periods, where their convective companion stars do not fill their Roche lobes thus reducing the mass accretion rates.

Table 2 summarizes the various source types of XRBs in the GC, including VFXTs. Only in the central 10 pc region, where the Swift-XRT sensitivity is high enough for detecting VFXTs down to $L_{X} \sim 10^{34} \mathrm{erg} \mathrm{s}^{-1}$, all 5 VFXTs have $\lesssim 10$ yr recurrence time on average. The 4 VFXTs located outside of the central 10 pc have each been detected in outburst once. It is uncertain if that is due to an intrinsically longer recurrence time for that population, or simply an observational bias effect resulting from decreased sensitivity.

Alternatively, transient intermediate polars (IPs) could account for a fainter population of VFXTs $\left(L_{\mathrm{X}} \sim\right.$ $10^{34} \mathrm{erg} \mathrm{s}^{-1}$ ) such as XMMU J175035.2-293557, which was discovered in the Galactic Bulge (Hofmann et al. 2018). However, the 9 VFXTs in the GC are not likely IPs since their X-ray outburst $L_{\mathrm{X}}$ increased by a factor of over 100, compared to this highly variable $(\sim 20 \times)$ IP. The nature of the VFXTs in the GC, whether they are ultra-compact XRBs (with a BH or NS) or NS-LMXBs with truncated accretion disks, remains an open question, at least unless/until their orbital periods are measured or type I X-ray bursts are detected during future recurrent X-ray outbursts. An ongoing Swift-XRT survey of the Galactic Bulge with follow-up optical/IR observations suggested that XRBs with evolved donors may also contribute to the VFXT population (Shaw et al. 2020).

\section{SPATIAL DISTRIBUTION OF THE BLACK HOLE BINARIES}

In this section we report our investigation of the spatial distribution of BHBs in the GC. We amalgamated the 12 quiescent, non-thermal X-ray sources detected by our Chandra data analysis (§2); two Swift transients from 2016 (Mori et al. 2019); the transient CXOGC J174540.0-290031 (Muno et al. 2005a); and the transient 1A 1742-289 (Davies et al. 1976), for a total of 16 BH-LMXB candidates (BHCs hereafter). In this section, we assume that all 12 non-thermal X-ray sources are BHCs. Nevertheless, we also performed the same analysis and present the results by excluding the three sources with no significant $(>90 \% \mathrm{CL}$ ) variability detection. All errors quoted in this section correspond to $1-\sigma$ uncertainty.

\subsection{Spatial fitting of the black hole binary distribution}

The 16 BHCs are projected on the 2-8 keV Chandra ACIS-I image in the left panel of Figure 6. The mean centroid of the $16 \mathrm{BHCs}$ is offset toward the south-east direction from $\mathrm{Sgr} \mathrm{A}^{*}$ by $\Delta \mathrm{RA}=0.8^{\prime \prime}$ and $\triangle \mathrm{DEC}=-7.6^{\prime \prime}$. The overall spatial distribution seems to be elongated along the south-east direction. First, we employed a linear regression to the positions of the 16 BHCs to characterize their 2D spatial distribution. Our linear regression fit yields a $-8 \pm 3^{\prime \prime}$ DEC offset from Sgr A* and a $38_{-9}^{+7}[\mathrm{deg}]$ position angle (defined relative to the north pole in the equatorial coordinate system). 

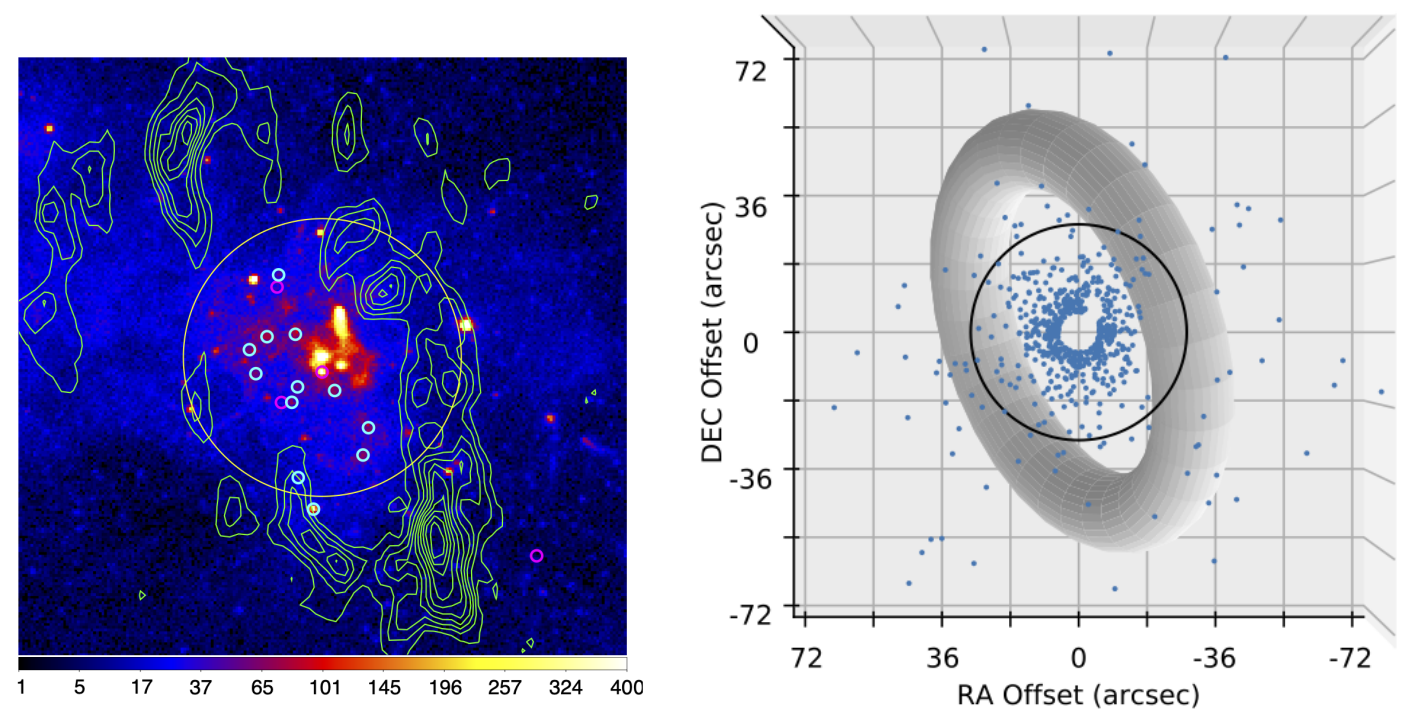

Figure 6. Left: $2-8 \mathrm{keV}$ Chandra ACIS-I image centered on Sgr A* of the 12 quiescent BH-LMXBs (cyan) and four BH transients (i.e., SXOTs, magenta) with CND contours (green). Right: A plot of 1,000 simulated sources (blue) overlaid with the 3D CND torus is shown to illustrate their spatial distribution and obscuration. Note that the regions at $r<0.2 \mathrm{pc}$ from Sgr A* and overlapping the bright diffuse X-ray sources (as shown in the left panel) are devoid of simulated sources. Also, some of the simulated sources inside/behind the CND are removed as they are not observable due to the significant X-ray absorption. The radius of the circle around Sgr A* in each figure is 1 pc, corresponding to $r=25^{\prime \prime}$.

Then, we fit a 2-D Gaussian profile

$$
N(x, y)=e^{a\left(x-x_{0}\right)^{2}-b\left(x-x_{0}\right)\left(y-y_{0}\right)-c\left(y-y_{0}\right)^{2}}
$$

where

$$
\begin{aligned}
& a=\cos ^{2}(\theta) / 2 \sigma_{x}^{2}+\sin ^{2}(\theta) / 2 \sigma_{y}^{2} \\
& b=\sin (2 \theta) / 2 \sigma_{x}^{2}-\sin (2 \theta) / 2 \sigma_{y}^{2} \\
& c=\sin ^{2}(\theta) / 2 \sigma_{x}^{2}+\cos ^{2}(\theta) / 2 \sigma_{y}^{2}
\end{aligned}
$$

to the 16 source positions, using the maximum likelihood estimation (MLE) method. $\theta$ is the position angle. $\left(x_{0}, y_{0}\right)$ are the coordinates of the centroid of the Gaussian ellipse as offset from Sgr A*. $\sigma_{x}$ and $\sigma_{y}$ are the widths along the major and minor axis, respectively. The best-fit centroid $\left(\Delta \mathrm{RA}=1 \pm 3^{\prime \prime}\right.$ and $\left.\Delta \mathrm{DEC}=-8 \pm 3^{\prime \prime}\right)$ is nearly consistent with the position of Sgr A*. The best-fit position angle $(\theta)$ is $41_{-10}^{+11}$ [deg]. These values are consistent with the linear regression results. The widths along the semi-major and semi-minor axis are $17^{\prime \prime+5}{ }_{-4}$ and $7_{-1}^{\prime \prime 2}$, respectively, yielding the eccentricity $\left(e \equiv \sqrt{1-\sigma_{y}^{2} / \sigma_{x}^{2}}\right)$ of $e=0.91_{-0.20}^{+0.05}$. Fitting to only the 9 sources with variability above $90 \%$ CL yields all parameter values within error of these reported values. We fit the 16 sources to a de-projected 3D radial power-law profile, $n(r)=k r^{-\gamma}$. The best-fit values are $k=0.13_{-0.06}^{+0.13} \operatorname{arcsec}^{-2}$ and $\gamma=2.3_{-0.2}^{+0.3}$.

The NSC is well-aligned with the Galactic Plane and therefore has a position angle of $31.4^{\circ} \pm 0.1^{\circ}$ (Schödel et al. 2014). On the other hand, the position angle of the clockwise young stellar disk in the central parsec is $99^{\circ} \pm 3^{\circ}$ (Bartko et al. 2009; Lu et al. 2009). Hence, the spatial distribution of the 16 BHCs is aligned with the NSC but not with the young stellar disk. In the next sections, we will investigate whether the disk-like distribution is induced by some observational biases.

\subsection{Observational systematics due to the circumnuclear disk and bright diffuse X-ray emission}

In the central few parsecs region, the circumnuclear disk (CND) and bright diffuse X-ray emission from the pulsar wind nebula (PWN) G359.95-0.04, Sgr A* and IRS 13 may inhibit observation of the intrinsic population distribution of BH-LMXBs. The hydrogen column density measurements of the CND in the radio and X-ray bands vary significantly from $N_{\mathrm{H}} \sim 10^{23}$ to $10^{25} \mathrm{~cm}^{-2}$ (Christopher et al. 2005; Mossoux \& Eckart 2018). The lower end of the CND column 
density $\left(N_{\mathrm{H}} \sim 10^{23} \mathrm{~cm}^{-2}\right)$ is comparable to the typical $N_{\mathrm{H}} \sim(1-2) \times 10^{23} \mathrm{~cm}^{-2}$ measured for the X-ray transients in the central parsec. On the other hand, if $N_{\mathrm{H}} \gtrsim 10^{25} \mathrm{~cm}^{-2}$, our XSPEC simulations show that an X-ray source with similar fluxes and spectral shapes as the observed quiescent BH-LMXBs will be completely absorbed in the Chandra energy band.

In order to account for these observation biases, we adopted the most conservative assumption that any X-ray sources inside/behind the CND or overlapping with the bright diffuse emission are undetectable by Chandra. Based on the geometry presented in Zhao et al. (2016), we modelled the CND as a 3-dimensional torus with an inclination angle of $61^{\circ}$ and a "roll" angle of $31^{\circ}$ to reflect the observed $19^{\circ}$ position angle of the CND's semi-major axis (see the right panel of Figure 6 for the CND geometry).

\subsection{Monte Carlo simulation}

We employed Monte Carlo (MC) simulations to test the hypothesis that the observed population of 16 BHCs represents an intrinsically spherical population centered at Sgr A*. Each simulation run generated a number of point sources using a spherically symmetric distribution with a 3D radial power-law profile of $n(r) \sim r^{-\gamma}$ with $\gamma=2.3$ extending from $r_{\min }=0.2 \mathrm{pc}$ to $r_{\max }=2.1 \mathrm{pc}$. A $\gamma$ value was updated from the $16 \mathrm{BHCs}$, which is different from that of H18 $(\gamma=2.4) \cdot r_{\min }=0.2 \mathrm{pc}$ was chosen as X-ray point sources cannot be detected within $0.2 \mathrm{pc}$ of Sgr A* due to its bright diffuse emission. $r_{\max }=2.1 \mathrm{pc}$ corresponds to the most distant BHC. Each simulated source was assigned 3D coordinates with respect to Sgr A*. We discarded sources that are located inside the CND or behind its line-of-sight. We also filtered out sources that overlap with Sgr A*, IRS13, PWN G359.95-0.04, and other diffuse emission features apparent in the Chandra image. In each MC realization, we generated fake sources so that we ended up with a number of "surviving" sources $\left(N_{S}\right.$ hereafter) similar to the observed number (i.e. 16 BHCs within $r \lesssim 2 \mathrm{pc}$ ).

After taking into account the observational systematics described above, we fit a 2-D gaussian function, described in equation (1), to the "surviving" simulated sources projected on the $2 \mathrm{D}$ sky plane. The best-fit position angle $(\theta)$ and eccentricity $(e)$ from each MC realization were determined using the same MLE method as applied to the 16 BHCs. This simulation and fitting process was iterated 10,000 times to construct an empirical probability distribution of the position angle and eccentricity.

As a result of the MC simulation, we observed that $\sim 37 \%$ of the simulated sources inside $r=2 \mathrm{pc}$ are obscured either by the CND or the bright diffuse emission. Most of the simulated sources, generated from a spherical distribution, exhibit distinct deviations from the observed orientation of the 16 BHCs. The distribution of position angles of the simulated X-ray sources is nearly uniform, as predicted from an assumed spherical distribution with some obscuration effects. The number of sample MC runs that fall within $1-\sigma$ of the best-fit position angle of the 16 BHCs $\left(\theta=41_{-10}^{+11}\right.$ $\left.\left[^{\circ}\right]\right)$ is 1287 out of 10,000 realizations $(\sim 13 \%)$. Therefore, we conclude that the observed BHCs are not consistent with a spherical distribution at $87 \%$ confidence level. On the other hand, we found that the distribution of eccentricity is highly dependent on $N_{S}$. When $N_{S}$ is large ( $\gtrsim 100$ ), we found that the eccentricity distribution was peaked at $e=0$ and decreases toward $e=1$, in line with the predicted outcome from spherical source distribution. In contrast, a smaller number of $N_{S}(\lesssim 20)$ led to a distribution skewed toward a higher $e$ value (e.g., $e=1$ in the most extreme case when $N_{S}=2$ ). Therefore, due to the limited number of observed samples (i.e. 16 BHCs) biasing the eccentricity distribution, we did not investigate the elongation of the source distribution using $e$.

Alternatively, we conducted a KS test to investigate whether the BHC distribution is statistically consistent with a spherically symmetric population. The KS test is non-parametric thus it is complementary to the 2D gaussian fit described above. First, we converted the 16 BHB positions to polar coordinates $(r, \theta)$ around Sgr A* and computed the cumulative distribution of their 16 position angles $(\theta)$. Second, we constructed a cumulative distribution function $(\mathrm{CDF})$ from the observable point sources in the above MC simulation; this serves as a model CDF. We found that 10,000 MC-simulated point sources deviate slightly from the uniform distribution over $\theta$ due to the obscuration and absorption effects in the GC. The model CDF was fit to an analytic function so that it could be implemented as an input to the KS test. As a result, a KS test on the actual CDF of 16 BHCs and the model CDF yields $K=0.28$ corresponding to a p-value of 0.11. Both the parametric and non-parametric tests suggest that the hypothesis of a spherical distribution is rejected at $\sim 90 \% \mathrm{CL}$.

Our further simulations show that we need at least 20 more BHCs in the central $1 \mathrm{pc}$ to firmly establish whether BHCs are distributed around Sgr $\mathrm{A}^{*}$ like a disk at $>3 \sigma$ level using their PA measurements. Future detection of $\mathrm{BH}$ transients by Swift and identifying more quiescent BH-LMXBs from the archived Chandra ACIS-S data analysis will enhance the number of BH-LMXBs in the central parsec region and allow us to determine the spatial distribution of the 
BHB cusp more accurately. The Lynx X-ray observatory, given its sub-arcsecond angular resolution and significantly larger effective area than Chandra, will be best suited for detecting and identifying a large number of fainter qXRBs in the GC.

\section{DISCUSSION}

Both the spatial and luminosity distributions of the 16 BHCs have several important implications for the recent $\mathrm{XRB}$ formation models in the GC as described below.

\subsection{Spatial distribution of BH binaries}

As shown in Figure 4 and 6, it is apparent that most of the 16 BHCs are concentrated in the central 1 pc, while the six NS-LMXBs are spread out over $\sim 50$ pc from the GC. Note that Bortolas et al. (2017) predicted that most NS should escape to outside the NSC due to their natal kicks (Bortolas et al. 2017). BH and WD are well populated within the NSC but the BH distribution is more cusp-like near the GC due to mass segregation, with a 3D power-law slope of $\gamma=1.72 \pm 0.04$ at $r<1.4$ pc whereas $\gamma=1.00 \pm 0.03$ for WD (Panamarev et al. 2019). These pictures are consistent with the high concentration of 16 BHCs inside $r \lesssim 1$ pc. The updated power-law slope of the observed BHC distribution $\left(\gamma=2.3_{-0.2}^{+0.3}\right)$ is close to the model prediction $(\gamma=1.72 \pm 0.04)$. Furthermore, Tagawa et al. (2020) demonstrated that gas-capture binaries (i.e. binaries formed by dissipating the kinetic energy in a dense gas) can reproduce the concentration of the $16 \mathrm{BHCs}$, by applying their AGN disk model to binary formation in the Galactic Center.

Although it is still only marginally significant, the disk-like distribution of the 16 BHCs has implications for recent theoretical predictions. Szölgyén \& Kocsis (2018) predicted that the isolated BH distribution should be elongated due to vector resonant relaxation. In this model, there is no specific orientation of the BH disk but it is probably determined by the latest episode of star formation in the GC. Our results indicate that the 16 BHCs are aligned with the NSC. Since the XRB formation in the GC follows the capture of companion stars in the NSC, the BH-LMXB distribution may track the NSC closely (Generozov et al. 2018; Panamarev et al. 2019). Using the resonance friction mechanism developed by Rauch \& Tremaine (1996), Gruzinov et al. (2020) also found that the distribution of stellar-mass BHs should be elongated and aligned with a rotating star cluster around the supermassive $\mathrm{BH}$.

\subsection{Number of BH-LMXBs in the central parsec region}

By extending the updated X-ray luminosity function of the 12 BHCs to a lower $L_{\mathrm{X}}$ value and using the best-fit slope $\alpha=1.3 \pm 0.1$ (where $N\left(>L_{X}\right) \propto L_{X}^{-\alpha}$ ), we can estimate the total number of qBH-LMXBs in the central parsec region. Note that the quiescent $L_{X}$ upper limits of the 2016 Swift transients (i.e., two of the four SXOTs) indicate that fainter BH-LMXBs exist in the central parsec below $L_{X} \approx 2 \times 10^{31} \mathrm{erg} \mathrm{s}^{-1}$ (Mori et al. 2019). It is apparent that the number estimate is most sensitive to the uncertainty in $\alpha$, and especially $L_{\min }$. By extending the best-fit X-ray luminosity function to $L_{\min }=2 \times 10^{31} \mathrm{erg} \mathrm{s}^{-1}$, we set the minimum number of BH-LMXBs to $\sim 45$ for $\alpha=1.3$ or $\sim$ 40-50 by allowing the range of $\alpha=1.2-1.4$. The number of BH-LMXBs increases if we adopt a previously reported luminosity distribution of local BH-LMXBs. Among the quiescent BH-LMXBs and their X-ray luminosities listed in Armas Padilla et al. (2014), the faintest BH-LMXB (SWIFT J1357.2-0933, $L_{\mathrm{X}}=8 \times 10^{29} \mathrm{erg} \mathrm{s}^{-1}$ ) may belong to a distinct group of BH-VFXTs and thus possibly follow a different luminosity function. Also, its distance uncertainty may lead to a higher quiescent X-ray luminosity of $L_{\mathrm{X}}=1.3 \times 10^{31} \mathrm{erg} \mathrm{s}^{-1}$ (Armas Padilla et al. 2014). Excluding the potential outlier SWIFT J1357.2-0933, the other BH-LMXBs in the Galactic plane have quiescent $L_{X}$ values above $\sim 3 \times 10^{30} \mathrm{erg} \mathrm{s}^{-1}$. By adopting $L_{\min }=3 \times 10^{30} \mathrm{erg} \mathrm{s}^{-1}$, we deduce that $\sim 500-630$ BH-LMXBs should populate the central parsec. However, if the three sources with no significant variability detection are rMSPs, the number of BH-LMXBs will be reduced to $\sim 240-300$. These numbers, which we consider as the most accurate estimate, are lower than those in H18 ( 600-1,000 and $\sim 450-750$ BH-LMXBs, respectively) since H18 adopted $L_{\text {min }}=8 \times 10^{29}$ erg s $^{-1}$ from Armas Padilla et al. (2014) as well as a different $\alpha$ value of 1.8 .

Panamarev et al. (2019) predicted that $\sim 6,000$ isolated BHs should reside inside 1.4 pc after 5 Gyrs and that the fraction of binaries should reach $\sim 2.5-5 \%$, indicating $\sim 150-300 \mathrm{BH}$ binaries. Generozov et al. (2018) predicted that 60-200 BH binaries should be formed by tidal capture. These numerical results are roughly consistent with the number of BH-LMXBs estimated from the measured luminosity function of the 12 non-thermal sources. Also, as discussed in $\S 3.4$, some of the VFXTs may represent a fainter population of BH-LMXBs (with orbital periods shorter than a few hours) which would increase the number of $\mathrm{BH}$ binaries in our estimates. It is thus imperative to identify the nature of VFXTs in the GC and detect fainter, quiescent BH-LMXBs in the solar neighborhood. 


\subsection{Population of close BH binaries in the Galactic Center}

Our systematic studies of the X-ray outburst history suggest that the 16 BHCs should have orbital periods in the range of $\sim 4-12 \mathrm{hrs}$. The N-body simulation of Panamarev et al. (2019) predicts that the binary separation (i.e. semi-major axis of binaries) is skewed toward $a \sim 0.01[\mathrm{AU}]$ after 5 Gyrs since the close binary systems have a higher chance of surviving against gravitational collisions with other stars in the NSC or 3-body interaction with the SMBH at Sgr A*. The binary separation between two stars with mass $m_{1}$ and $m_{2}$ is given by $a=2.3 \times 10^{-3}\left(m_{1}+m_{2}\right)^{1 / 3} P_{\text {orb }}^{2 / 3}$ [AU] where $P_{\text {obs }}$ is an orbital period [hrs]. In $\S 3.3$, we argued that the 16 BHCs should have $P_{\text {orb }} \sim 4-12$ hrs. For a 10 $M_{\odot} \mathrm{BH}$ binary with $P_{\mathrm{orb}}=4-12 \mathrm{hrs}, a \sim 0.01-0.03$ [AU]. This range is consistent with the semi-major axis distance distribution of binaries in the inner NSC region as derived from the N-body simulation (Panamarev et al. 2019). In addition, Generozov et al. (2018) also argued that XRBs should have orbital periods shorter than $\sim 10$ hours if they are formed via tidal capture around Sgr A*. Both our study of X-ray sources in the GC and the recent theoretical models for XRB formation around Sgr A* agree that BH binaries in the central parsec should be tightly bound with $P_{\text {orb }} \lesssim 12$ hours. If future X-ray observations reveal that some of the VFXTs in the GC are ultra-compact XRBs with $P_{\text {orb }}$ shorter than a few hours, it will support this picture even further.

\section{SUMMARY}

- Our subsequent analysis of the 3 Msec Chandra ACIS-S/HETG and 1.6 Msec ACIS-S data from 2012-2018 confirmed 11 of the dozen non-thermal X-ray sources from H18 (which was based on 1.4 Msec ACIS-I data) and detected an additional non-thermal source in the central parsec as a result of much improved statistics. We detected significant long-term variability from 9 of the 12 non-thermal sources, indicating that a majority of them are quiescent XRBs rather than rMSPs. It is still possible that one and three of the dozen non-thermal sources may be an SS Cyg-like CV or rMSPs, respectively.

- Based on the most complete X-ray outburst data collected by frequent X-ray monitoring of the GC, we found that the 12 quiescent XRB candidates (with no outburst detected) as well as the four X-ray transients with single outbursts are distinct from the six NS-LMXBs and five VFXTs (within $r \lesssim 10$ pc) which display recurrent X-ray outbursts.

- The 16 non-recurring XRBs - 12 quiescent, non-thermal sources and 4 SXOTs - are likely BH-LMXBs with an orbital period range of $P_{\text {orb }} \sim 4-12$ hours, based on their outburst history and quiescent X-ray properties as inferred from the recent studies of X-ray outbursts by Lin et al. (2019) and Wu et al. (2010).

- The spatial distribution of the 16 BH-LMXB candidates is consistent with a disk-like distribution aligned with the NSC. A spherical distribution is marginally rejected at a the $\sim 90 \%$ level.

- An updated X-ray luminosity function yields the number of BH-LMXBs in the central parsec $\sim 500-630$, or $\sim 240-300$ if the three sources with no significant variability detection are rMSPs. These estimates are roughly consistent with the predictions by the theoretical model and N-body simulation employed by Generozov et al. (2018) and Panamarev et al. (2019), respectively.

- While only six outbursting NS-LMXBs have been identified through type I X-ray bursts within $r \lesssim 50 \mathrm{pc}$, a larger number of quiescent NS-LMXBs may exist in the GC as observed in globular clusters (Heinke et al. 2003). Those faint NS-LMXBs with mostly soft thermal X-ray emission $(k T \lesssim 0.1 \mathrm{keV})$ would remain undetected as their X-ray emission is heavily obscured by the large hydrogen column density $\left(N_{\mathrm{H}} \sim 10^{23} \mathrm{~cm}^{-2}\right)$ in the GC, and they do not show X-ray outbursts (not even faint ones) due to their low accretion rates.

- We derived a binary separation range of $a \sim 0.01-0.03$ [AU] of the BH-LMXB population in the central parsec, assuming that a majority of them have $P_{\text {orb }} \sim 4-12$ hours and $10 M_{\odot} \mathrm{BH}$ mass. These results provide direct evidence for the predictions of Generozov et al. (2018) and Panamarev et al. (2019). Both our results and their models suggest that XRBs in the central parsec should be tightly bound; otherwise, the gravitational interaction with the SMBH at Sgr A* and collisions with other stars in the NSC can destroy the binaries. 


\section{ACKNOWLEDGMENTS}

We thank Brian Metzger, Nicholas Stone, Aleksey Generozov, Barry McKernan, K.E. Saavik Ford and MordecaiMark Mac Low for discussions on the population of X-ray binaries and gravitational wave events in galactic nuclei. We thank Benjamin Vermette for simulating Chandra ACIS spectra of non-magnetic CVs, including SS Cyg. GP acknowledges financial contribution from the agreement ASI-INAF n.2017-14-H.0.

Software: HEASoft Version 6.27 (HEASARC 2014), FTools Version 6.27 (HEASARC 2014)

\section{REFERENCES}

Armas Padilla, M., Wijnands, R., Degenaar, N., et al. 2014, MNRAS, 444, 902

Arnaud, K. A. 1996, in Astronomical Society of the Pacific Conference Series, Vol. 101, Astronomical Data Analysis Software and Systems V, ed. G. H. Jacoby \& J. Barnes, 17

Arur, K., \& Maccarone, T. J. 2018, MNRAS, 474, 69

Bahcall, J. N., \& Wolf, R. A. 1976, ApJ, 209, 214

—. 1977, ApJ, 216, 883

Bahramian, A., Strader, J., Miller-Jones, J. C. A., et al. 2020, ApJ, 901, 57

Bartko, H., Martins, F., Fritz, T. K., et al. 2009, ApJ, 697, 1741

Baskill, D. S., Wheatley, P. J., \& Osborne, J. P. 2005, MNRAS, 357, 626

Baumgardt, H., Amaro-Seoane, P., \& Schödel, R. 2018, A\&A, 609, A28

Bogdanov, S., Grindlay, J. E., Heinke, C. O., et al. 2006, ApJ, 646, 1104

Bogdanov, S., Heinke, C. O., Özel, F., \& Güver, T. 2016, ApJ, 831, 184

Bortolas, E., Mapelli, M., \& Spera, M. 2017, MNRAS, 469, 1510

Broos, P. S., Townsley, L. K., Feigelson, E. D., et al. 2010, ApJ, 714, 1582

Camarillo, T., Mathur, V., Mitchell, T., \& Ratra, B. 2018, PASP, 130, 024101

Carbone, D., \& Wijnands, R. 2019, MNRAS, 488, 2767

Christopher, M. H., Scoville, N. Z., Stolovy, S. R., \& Yun, M. S. 2005, ApJ, 622, 346

Conner, J. P., Evans, W. D., \& Belian, R. D. 1969, ApJL, 157, L157

Coriat, M., Fender, R. P., \& Dubus, G. 2012, MNRAS, 424, 1991

Corral-Santana, J. M., Casares, J., Muñoz-Darias, T., et al. 2016, A\&A, 587, A61

Davies, R. D., Walsh, D., Browne, I. W. A., Edwards, M. R., \& Noble, R. G. 1976, Nature, 261, 476

Degenaar, N., Patruno, A., \& Wijnands, R. 2012a, ApJ, 756,148
Degenaar, N., \& Wijnands, R. 2009, A\&A, 495, 547

Degenaar, N., Wijnands, R., Cackett, E. M., et al. 2012b, A\&A, 545, A49

Degenaar, N., Wijnands, R., Miller, J. M., et al. 2015, Journal of High Energy Astrophysics, 7, 137

Fragione, G., Grishin, E., Leigh, N. W. C., Perets, H. B., \& Perna, R. 2019, MNRAS, 488, 47

Gallo, E., Degenaar, N., \& van den Eijnden, J. 2018, MNRAS, 478, L132

Garcia, M. R., McClintock, J. E., Narayan, R., et al. 2001, ApJL, 553, L47

Generozov, A., Stone, N. C., Metzger, B. D., \& Ostriker, J. P. 2018, MNRAS, 478, 4030

Gruzinov, A., Levin, Y., \& Zhu, J. 2020, ApJ, 905, 11

Hailey, C. J., Mori, K., Bauer, F. E., et al. 2018, Nature, 556,70

Hailey, C. J., Mori, K., Perez, K., et al. 2016, ApJ, 826, 160

Hameury, J., \& Lasota, J. 2016, A\&A, 594, A87, 9pp

HEASARC. 2014, HEAsoft: Unified Release of FTOOLS and XANADU, ascl:1408.004

Heinke, C. O., Bahramian, A., Degenaar, N., \& Wijnands, R. 2015, MNRAS, 447, 3034

Heinke, C. O., Grindlay, J. E., Lloyd, D. A., \& Edmonds, P. D. 2003, ApJ, 588, 452

Hofmann, F., Ponti, G., Haberl, F., \& Clavel, M. 2018, A\&A, 615, L7

Hong, J., Mori, K., Hailey, C. J., et al. 2016, ApJ, 825, 132

Jin, C., Ponti, G., Haberl, F., Smith, R. a., \& Valencic, L. 2018, MNRAS, 477, 3480

Kennea, J. A., Burrows, D. N., Kouveliotou, C., et al. 2013, ApJL, 770, L24

Knevitt, G., Wynn, G. A., Vaughan, S., \& Watson, M. G. 2014, MNRAS, 437, 3087

Lin, J., Yan, Z., Han, Z., \& Yu, W. 2019, ApJ, 870, 126

Lu, J. R., Ghez, A. M., Hornstein, S. D., et al. 2009, ApJ, 690, 1463

Maccarone, T. J., \& Patruno, A. 2012, MNRAS, 428, 1335

Maeda, Y., Koyama, K., Sakano, M., Takeshima, T., \& Yamauchi, S. 1996, PASJ, 48, 417 
Matsuoka, M., Inoue, H., Koyama, K., et al. 1980, ApJL, 240, L137

McKernan, B., Ford, K. E. S., Bellovary, J., et al. 2018, ApJ, 866, 66

Miller, M. C., \& Miller, J. M. 2015, PhR, 548, 1

Miralda-Escudé, J., \& Gould, A. 2000, ApJ, 545, 847

Mori, K., Gotthelf, E. V., Zhang, S., et al. 2013, ApJL, 770, L23

Mori, K., Hailey, C. J., Mandel, S., et al. 2019, ApJ, 885, 142

Morris, M. 1993, ApJ, 408, 496

Mossoux, E., \& Eckart, A. 2018, MNRAS, 474, 3787

Mukai, K. 2017, PASP, 129, 062001

Muno, M. P., Lu, J. R., Baganoff, F. K., et al. 2005a, ApJ, 633, 228

Muno, M. P., Pfahl, E., Baganoff, F. K., et al. 2005b, ApJL, 622, L113

Muno, M. P., Arabadjis, J. S., Baganoff, F. K., et al. 2004, ApJ, 613, 1179

Muno, M. P., Bauer, F. E., Baganoff, F. K., et al. 2009, ApJS, 181, 110

Panamarev, T., Just, A., Spurzem, R., et al. 2019, MNRAS, 484, 3279

Perez, K., Hailey, C. J., Bauer, F. E., et al. 2015, Nature, 520,646

Plotkin, R. M., Gallo, E., \& Jonker, P. G. 2013, ApJ, 773, 59

Ponti, G., De, K., Muñoz-Darias, T., Stella, L., \& Nandra, K. 2017, MNRAS, 464, 840
Porquet, D., Grosso, N., Bélanger, G., et al. 2005, A\&A, 443, 571

Rauch, K. P., \& Tremaine, S. 1996, NewA, 1, 149

Rea, N., Esposito, P., Pons, J. A., et al. 2013, ApJL, 775, L34

Reynolds, M. T., Reis, R. C., Miller, J. M., Cackett, E. M., \& Degenaar, N. 2014, MNRAS, 441, 3656

Scargle, J. D., Norris, J. P., Jackson, B., \& Chiang, J. 2013, ApJ, 764, 167

Schödel, R., Feldmeier, A., Kunneriath, D., et al. 2014, A\&A, 566, A47

Shaw, A. W., Heinke, C. O., Maccarone, T. J., et al. 2020, MNRAS, 492, 4344

Sleator, C. C., Tomsick, J. A., King, A. L., et al. 2016, ApJ, 827, 134

Szölgyén, Á., \& Kocsis, B. 2018, Physical Review Letters, 121,101101

Tagawa, H., Haiman, Z., \& Kocsis, B. 2020, ApJ, 898, 25

van den Eijnden, J., Degenaar, N., Pinto, C., et al. 2018, MNRAS, 475, 2027

Walsh, A. R., Cackett, E. M., \& Bernardini, F. 2015, MNRAS, 449, 1238

Wu, Y. X., Yu, W., Li, T. P., Maccarone, T. J., \& Li, X. D. 2010, ApJ, 718, 620

Xu, X.-j., Li, Z., Zhu, Z., et al. 2019, ApJ, 882, 164

Xu, X.-j., Wang, Q. D., \& Li, X.-D. 2016, ApJ, 818, 136

Yan, Z., \& Yu, W. 2015, ApJ, 805, 87

Zhao, J.-H., Morris, M. R., \& Goss, W. M. 2016, ApJ, 817, 171

Zhu, Z., Li, Z., \& Morris, M. R. 2018, ApJS, 235, 26 